\title{
Safety Assessment of Rigid Frame Cantilever Construction Based on Matter-Element and Extension
}

\author{
Zhu Qinghua*, Tian Zhongchu \\ School of Civil Engineering, Changsha University of Science \& Technology, Changsha, China
}

Email address:

1123137456@qq.com (Zhu Qinghua)

*Corresponding author

\section{To cite this article:}

Zhu Qinghua, Tian Zhongchu. Safety Assessment of Rigid Frame Cantilever Construction Based on Matter-Element and Extension. Science Discovery. Vol. 9, No. 2, 2021, pp. 37-42. doi: 10.11648/j.sd.20210902.13

Received: March 8, 2021; Accepted: March 26, 2021; Published: March 30, 2021

\begin{abstract}
In order to effectively evaluate the safety status of the cantilever construction of a large-span rigid frame bridge, this paper takes a four-span rigid frame bridge as the engineering background, selects the failure probability of the cantilever construction, the natural environment, the cantilever construction operation, and the management level as the evaluation factors and divides the possibility level into Five levels, an extension model is established to evaluate the cantilever construction of the bridge. This paper first uses fuzzy comprehensive evaluation to quantify the natural environment and cantilever construction technology, then calculates the failure probability of the bridge cantilever construction based on Monte Carlo theory, and finally scores according to the management level of the management system. Invite relevant experts to obtain the weight set of each evaluation factor through the 1-9 scale method, and use the goodness evaluation method in the matter-element extension method for evaluation. The results show that the safety assessment result of the cantilever construction of the bridge is a medium risk, which provides a reliable basis for predicting and avoiding risks. Based on the matter-element extension method, the rigid frame cantilever construction safety assessment takes into account the uncertainty and ambiguity of failure, and more objectively reflects the safety status of the cantilever construction during the construction period. The calculation results of different safety evaluation models are compared with the results of this paper, which verifies the feasibility and effectiveness of the matter-element extension theory applied to the safety evaluation of cantilever construction.
\end{abstract}

Keywords: Fuzzy Comprehensive Evaluation, Extension Theory, Probability of Failure, Reliability

\section{基于物元可拓法刚构悬臂施工安全评估}

朱清华"，田仲初

长沙理工大学土木工程学院, 长沙, 中国

邮箱

1123137456@qq.com（朱清华）

摘要: 为了有效评估大跨度刚构桥悬臂施工安全状况, 本文以某四跨刚构桥为工程背景, 选择悬臂施工失效概率、自然 环境、悬臂施工工艺、管理水平作为评价因子并将风险-划分为五个等级, 建立可拓模型对该桥悬臂施工进行评估。本文 首先对自然环境与悬臂施工工艺采用模糊综合评价进行量化处理, 然后根据蒙特卡罗理论计算出大桥悬臂施工失效概率, 最后则根据管理系统管理水平进行打分。邀请相关专家通过1 9标度法得到每个评价因子的权重集，采用物元可拓法中 的优度评价法进行评估。结果表明, 该桥县臂施工安全评估结果为中等风险, 为预测和避免风险提供了可靠依据。基于 物元可拓法刚构悬臂施工安全评估考虑了失效的不确定性及模糊性, 较为客观地反应施工期间悬臂施工的安全状态。将 不同安全评估模型计算的结果与本文结果对比, 验证了物元可拓理论应用于悬臂施工安全评估的可行性与有效性。 
关键词：模糊综合评价, 可拓学理论, 失效可能性, 可靠度

\section{1. 引言}

悬臂施工法具有良好的经济性，因而成为跨越山谷、 河流或桥下净空受到限制时优先考虑的施工方法。但在桥 梁的施工期间，因自然环境、施工工艺不当或者管理不到 位等原因造成桥梁失效事故频发。针对结构可靠性的问题, 穆加宇提出了动态可靠度理论与“理想临界结构”相联系的 概念, 将可靠性划分为不同等级并定量计算出结构的可靠 性[1]。刘杨、张建仁在引入特殊的修正联合概率密度函数 的基础上, 将有限元法与退火进化算法相结合用以研究结 构的模糊可靠度 [2]。王磊基于模糊数学理论与 AHP 法 (层 次分析法) 把影响桥梁构件承载力不确定性的因素系统化, 利用模糊数学将定性描述定量化 [3]。岐峰军对参数离散性 下刚构桥稳定性可靠度分析方面进行了研究, 采用MC法产 生伪样本进行参数预测从可靠度出发准确评估刚构桥安全 性[4]。Davis-McDaniel 等人在结合了大量桥梁失效事故的 基础上，利用故障树建模分析桥梁安全风险[5]。

结构安全评估一般有两种方式,一是采用可靠度理论 对其进行失效概率分析, 二是采用模糊数学的理论对结构 风险进行模糊判断。但桥梁结构是一个复杂的大型系统, 对其可靠性产生影响的因素众多, 往往需要综合可靠度理 论与模糊数学的理论对其工作状况进行分析。但可靠度理

论与模糊数学往往用不同的表达方式, 难以有效综合两者 进行评价。蔡文创立可拓工程学能够描述事物的可变性, 将定性描述转变为定量描述, 并通过建立多指标的评估模 型来完整评价事物 [6]。杨雅勋应用可拓模型对某连续梁桥 进行状态评估[7]。张海提出将可拓理论应用于对混凝土梁 桥可靠性进行综合评价[8]。张延年运用多指标多级可拓评 价方法评价桥梁下部结构的总体可靠性[9]。

文献[7～9]借助可拓理论对成桥状态下结构可靠性进 行了有效评价, 但针对大跨度桥梁悬臂施工可靠性的研究 较少涉及。本文基于物元可拓理论建立评估模型, 对某刚 构桥悬臂施工安全进行评价, 以期望其更具客观性、合理 性, 为桥梁悬臂施工提供重要依据。

\section{2. 建立物元可拓评估模型}

可拓工程学中的物元可拓法主要用于处理不相容问 题, 解决了传统数学中“非此即彼”的局限, 能将定量分析 与定性分析统一起来, 并且能合理地反映评价对象状况 [10]。本文采用物元可拓的优度评价法评价所研究的对象, 首先确认评价指标, 然后确定各个指标的量域, 之后确认 各个关联函数。具体可拓评估流程见图1。

悬臂施工状态等级的确定

确定指标权重

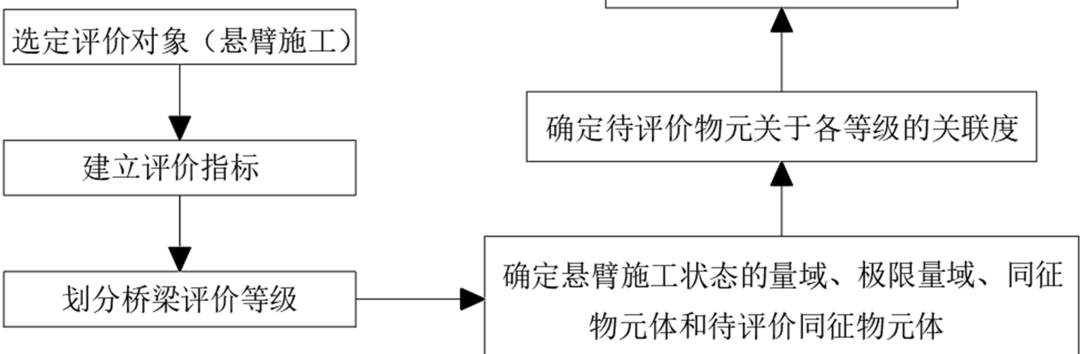

图1 可拓评估流程。

\section{1. 确认评价指标}

根据可拓理论的研究,待评估的对象失效可能性 $L$, 划 分为 $i$ 个等级: $L_{1}, L_{2}, L_{3}, \ldots, L_{i}$ 分别对应风险矩阵失效 可能性等级的 $1,2,3, \ldots i$, 物元如上所示为事物、特征 及事物的特征值构成, 可用 $R=$ (事物, 特征, 量值)三元 组表示,则待评价物的物元R如下。

$$
R=\left[\begin{array}{ccc}
L & c_{1} & v_{1} \\
& c_{2} & v_{2} \\
& c_{3} & v_{3} \\
\vdots & \vdots \\
c_{i} & v_{i}
\end{array}\right]
$$

式中 $c_{i}$ 为待评估所需衡量条件, $v_{i}$ 为待评估对象的综 合评价结果。

\section{2. 确认与评价等级相应的各指标的量域和极限量域}

用对应于每个失效等级 $L_{j}$ 的物元体来体现失效可能 性等级的量域 $V_{i j}$, 根据各个指标可能的取值范围, 可知极 限量域为 $V_{i 0} \circ R_{0 j}$ 和 $R_{0}$ 为单位标准物元 $[10]$ 。

$$
R_{0 j}=\left[\begin{array}{ccc}
L_{j} & c_{1} & V_{1 j} \\
& c_{2} & V_{2 j} \\
& c_{3} & V_{3 j} \\
& \vdots & \vdots \\
& c_{i} & V_{i j}
\end{array}\right]
$$




$$
R_{0}=\left[\begin{array}{ccc}
N_{0} & c_{1} & V_{10} \\
& c_{2} & V_{20} \\
& c_{3} & V_{30} \\
\vdots & \vdots \\
& c_{i} & V_{i 0}
\end{array}\right]
$$

\section{3. 确认关联函数}

在可拓评价中,优度评价法是评价对象的基本方法,在 优度评价中存在两个基本概念, 即合格度和优度。合格度 是指评价因素符合要求的程度, 而优度则是方案优劣的量 化值。

在确定待评物元之后, 利用关联函数可以计算各评价 等级关于某个衡量条件的合格度 [11]。失效可能性等级 $L_{j}$ 关于衡量条件即评价指标 $c_{i}$ 的合格度为 $K_{j}\left(v_{i}\right)$ 。

$$
\begin{gathered}
p\left(v_{i}, V_{i j}\right)=\left|v_{i}-\frac{a_{i j}+b_{i j}}{2}\right|-\frac{b_{i j}-a_{i j}}{2} \\
p\left(v_{i}, V_{i 0}\right)=\left|v_{i}-\frac{a_{i 0}+b_{i 0}}{2}\right|-\frac{b_{i 0}-a_{i 0}}{2} \\
D\left(v_{i}, V_{i j}, V_{i 0}\right)=p\left(v_{i}, V_{i 0}\right)-p\left(v_{i}, V_{i j}\right) \\
K_{j}\left(v_{i}\right)=\frac{p\left(v_{i}, V_{i j}\right)}{D\left(v_{i}, V_{i j}, V_{i 0}\right)}
\end{gathered}
$$

为方便对评价对象的优劣进行比较, 需要将合格度进 行规范化。设评价对象的具体取值为 $I$ 为 $K(I)$, 对 $K(I)$ 进 行规范化。

假设符合条件的量值范围为量域 $V_{0}$, 量值允许取值的 范围为极限量域为 $V$, 设 $v$ 为 $V$ 上任意取值或者区间。

当 $v \notin V_{0}$ 时, $D\left(v, V_{0}, V\right)$ 的取值为 $\rho\left(v, V_{0}\right)-\rho\left(v_{0}, V_{0}\right)$; 当 $v \in V_{0}$ 时, $D\left(v, V_{0}, V\right)$ 的取值为 -1 。

$$
k_{I}=\left\{\begin{array}{cc}
\frac{K(I)}{\max _{v \in V_{0}} K_{i}(v)} & K(I)>0 \\
\frac{K(I)}{\max _{V \notin V_{0}}\left|K_{i}(v)\right|} & K(I)<0
\end{array}\right.
$$

\section{4. 确认权系数}

对于失效可能性评价的 $i$ 个评价指标，采用邀请相关 专家通过1 9标度法 (见表1), 对各评价指标进行两两比 较并赋值, 构造成判断矩阵并计算权重[12]。首先建立判 断矩阵:

$$
R=\left[\begin{array}{ccc}
1 & \cdots & a_{1 i} \\
\vdots & \ddots & \vdots \\
1 / a_{1 i} & \cdots & 1
\end{array}\right]
$$

计算判断 $R$ 中每一行元素的乘积 $M_{i}$, 再求 $M_{i}$ 的 $n$ 次方根 从得到 $\bar{A}_{i}=\sqrt[n]{M_{n}}$, 对 $\bar{A}_{i}$ 进行归一化处理, 获得权系数:

$$
A=\left[a_{1}, a_{2}, a_{3}, \ldots a_{i}\right]
$$

\section{5. 计算优度}

在得到各个评价等级关于评价指标的规范合格度后, 结合权系数, 可计算出各等级的优度。

$$
C\left[L_{i}\right]=A K\left(L_{i}\right)=\left[a_{1}, a_{2}, a_{3}, \ldots a_{i}\right]\left[\begin{array}{c}
k_{1 i} \\
k_{2 i} \\
k_{3 i} \\
\vdots \\
k_{j i}
\end{array}\right]
$$

对各等级的优度大小进行比较, 优度越大, 与某等级

\begin{tabular}{|c|c|c|c|}
\hline 标度 & 含义 & 标度 & 含义 \\
\hline 1 & 表示两个因素同等重要 & 7 & 表示一个因素比另一个因素强烈重要 \\
\hline 3 & 表示一个因素比另一个因素稍微重要 & 9 & 表示一个因素比另一个因素极端重要 \\
\hline 5 & 表示一个因素比另一个因素明显重要 & $2 、 4 、 6 、 8$ & 表示上诉相邻判断的中值 \\
\hline \multicolumn{4}{|c|}{ 若因素 $i$ 与因素 $j$ 比较标度为 $a_{i j}$, 则因素 $j$ 与因素 $i$ 的标度为 $1 / a_{i j}$} \\
\hline
\end{tabular}
的符合程度就越高。

\section{3. 工程实例验算}

以某连续刚构桥为工程背景, 大桥为四跨连续刚构桥, 主跨跨径 $120 \mathrm{~m}$, 大桥平面位于圆曲线上, 未设置纵坡。 桥梁主梁采用C55混凝土悬臂浇筑施工, 主梁预应力采用 三向预应力系统，包含纵向预应力束、横向预应力束和坚 向预应力束。

表1 1-9标度法。

\section{1. 确定评价等级及评价指标} 如下:

首先建立评估模型指标, 各评价指标的各区间值确定

大桥失效概率取值为 $(0,1)$, 参考国际隧道协会颁布风 险概率描述, 分为五个子区间 $\left(0,10^{-4}\right),\left(10^{-4}, 10^{-3}\right),\left(10^{-3}, 10^{-2}\right)$, $\left(10^{-2}, 10^{-1}\right),\left(10^{-1}, 1\right)$ 分别对应非常低、低、中、高、极高风 险 $[13]$ 。

自然环境和悬臂施工工艺均由模糊综合评价得出, 故 取值均为范围在 $(0,1)$ 的隶属度值。将 $(0,1)$ 区间进行子区 间划分, 分别划分为 $(0,0.2),(0.2,0.4),(0.4,0.6),(0.6,0.8)$, $(0.8,1)$ 。分别对应大桥失稳影响程度从低到高的 5 个等级。
表2 悬臂施工工艺评判矩阵 $\mathrm{B}$ 和各因素权重。

\begin{tabular}{llllllll}
\hline $\boldsymbol{B}_{\mathbf{1}}$ & 评价内容 & $\boldsymbol{b}_{\mathbf{1 1}}$ & $\boldsymbol{b}_{\mathbf{1 2}}$ & $\boldsymbol{b}_{\mathbf{1 3}}$ & $\boldsymbol{b}_{\mathbf{1 4}}$ & $\boldsymbol{b}_{\mathbf{1 5}}$ & $\boldsymbol{\omega}$ \\
\hline$b_{11}$ & 安装和浇 & 1 & 5 & 2 & 3 & $1 / 2$ & 0.305 \\
$b_{12}$ & 筑操作 & $1 / 5$ & 1 & 2 & 1 & $1 / 2$ & 0.129 \\
$b_{13}$ & 养护情况 & $1 / 2$ & $1 / 2$ & 1 & 2 & $1 / 3$ & 0.124 \\
$b_{14}$ & $\begin{array}{c}\text { 配重情况 } \\
\text { 挂篮情况 }\end{array}$ & $1 / 3$ & 1 & $1 / 2$ & 1 & $1 / 2$ & 0.108 \\
$b_{15}$ & $\begin{array}{l}\text { 预应力张 } \\
\text { 拉情况 }\end{array}$ & & 2 & 3 & 2 & 1 & 0.335 \\
$\lambda_{\max }$ & $=5.412 \quad C_{I}=0.103$ & $C_{R}=0.092<0.1$ & & \\
\hline
\end{tabular}

大桥管理水平根据风险检测（RBI）中的管理系统来 判断, 得分取值区间为 $(0,1000)$, 将区间按照管理水 
平对悬臂施工失效影响程度从低到高排列可得 5 个等级的 子区间 $(800,1000),(600,800),(600,400),(400,200),(0,200)$ 。

\section{2. 模糊综合评价}

对自然环境和悬臂施工工艺均采用模糊综合评价量化, 由于求解过程一样, 此处仅列出施工操作的模糊综合评价。

建立因素集即影响评价对象的各种因素的集合。对于 悬臂施工工艺的评价因素集可表示为 $\{u\}$, 其中包含安装 和浇筑操作、养护情况、预应力张拉情况、挂篮情况、配 重情况。根据各因素施工现场对桥梁影响的程度及发生后 果的严重程度, 将各因素分为五个等级, 即评价集 $\mathrm{V}=\{\mathrm{I}$ 级、II 级、III级、 IV 级、 $\mathrm{V}$ 级 $\}=\{$ 优, 良, 中, 合格、差 $\}$ 。 邀请相关专家通过 $1 \sim 9$ 标度法对各因素间进行两两比较 并赋值, 构造成判断矩阵并计算权重, 上面是施工操作的 判断矩阵 $B$ 和权重（如上表 2 所示）。

考虑到专家们根据工程经验和专业知识判断因素之 间的相对重要程度, 其各因素的赋值会受到主观因素的影 响, 评价结果的准确性难以得到保证; 因此需要对判断矩 阵进行一致性的检验, 最终才能得到其指标的评价结果。 由各专家通过评价集（表2）对各因素的指标进行打分,采 用梯形隶属度函数确定施工操作的评价矩阵 $R$, 结合权重 $\omega$ 得到一级评价矩阵向量 $C$, 即 $C=\omega \cdot R$ 。

$$
R=\left[\begin{array}{ccccc}
0.5 & 0.5 & 0 & 0 & 0 \\
0 & 0.8 & 0.2 & 0 & 0 \\
0.2 & 0.8 & 0 & 0 & 0 \\
1 & 0 & 0 & 0 & 0 \\
0.5 & 0.5 & 0 & 0 & 0
\end{array}\right]
$$

$$
C=\omega \cdot R=\left\{\begin{array}{llll}
0.452, & 0.522, & 0.026,0,0
\end{array}\right\}
$$

依据模糊数学的最大隶属度原则, 其评价为 0.522 , 将该桥的悬臂施工操作模糊评价为良的状态。对于自然环 境的评价因素集可表示为 $\left\{u_{1}\right\}$, 其中包含地质条件、地貌 条件、自然灾害发生频率, 同理自然环境的模糊评价为 0.485 , 状态为良。

\section{3. 量化评价指标}

参考可靠度理论, 通过Midas有限元软件选用关键截 面的样本结果, 将其输入Matlab拟合的连续刚构桥各关 键截面失效形式功能函数 $G(\hat{X})[15]$ 。
利用蒙特卡罗法计算大桥悬臂施工可靠度, 通过 Matlab生成出蒙特卡罗法可靠度计算的样本, 计算出每 一个样本的值, 对于小于零或等于零的为失效, 对每一个 样本都进行计算和判断, 统计失效的次数, 最后即可悬臂 施工结构体系失效概率:

$$
\hat{p}_{f}=\frac{1}{N} \sum_{i=1}^{N} I\left[G(\hat{X})_{i}\right]
$$

式中, $\mathrm{N}$ 为计算样本总数, $G(\hat{X})_{i}$ 值为非正数时, $I\left[G(\hat{X})_{i}\right]=1$; 对应的 $G(\hat{X})_{i}$ 为正数时, $I\left[G(\hat{X})_{i}\right]=0$, “^”为 计算样本标志, 函数分类函数。

本文通过Matlab选取样本 1200000 个, 计算失效 121 个, 失效概率为 $1.008 e^{-4}$ 。

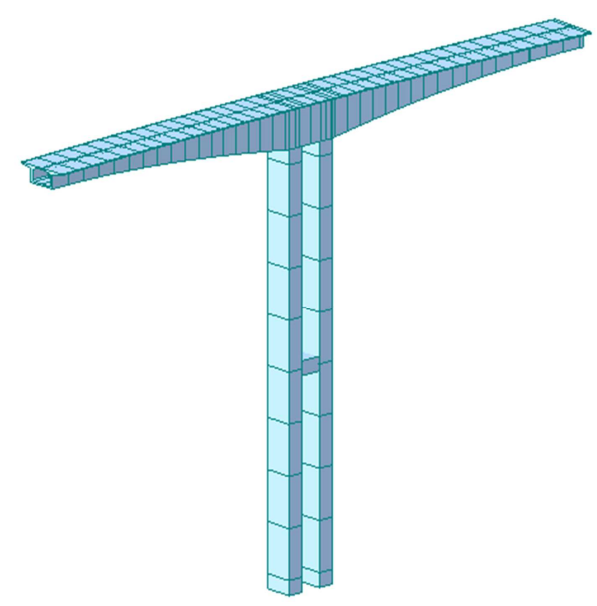

图2 最大悬臂阶段有限元模型图。

该桥悬臂施工工艺和自然环境均通过模糊综合评价 得出分别为 0.522 和 0.485 , 根据专家依据表 3 对管理水平进 行打分值, 该桥管理水平分数为 680 。待评价物元 $\boldsymbol{R}$ 表示为

$$
R=\left[\begin{array}{ccc}
L & c_{1} & 1.008 e^{-4} \\
& c_{2} & 0.522 \\
& c_{3} & 0.485 \\
c_{4} & 680
\end{array}\right]
$$

计算待评价物元与各等级的关联度后, 对其进行规范 化处理, 即可得出规范合格度。

表3 $V_{4}$ 管理水平估计指标。

\begin{tabular}{llll}
\hline 指标 & 主题 & 考核问题数目 & 分数 \\
\hline 1 & 领导与管理 & 6 & 75 \\
2 & 工艺安全信息 & 11 & 85 \\
3 & 工艺危害分析 & 5 & 105 \\
4 & 检查制度 & 6 & 80 \\
5 & 技术交底 & 8 & 100 \\
6 & 人员配置 & 10 & 95 \\
7 & 紧急响应 & 13 & 80 \\
8 & 材料承包商 & 12 & 55 \\
9 & 培训 & 9 & 90 \\
10 & 施工机具完整 & 10 & 110 \\
11 & 运行规程 & 12 & 80 \\
12 & 审核 & 8 & 55 \\
& 总计 & 110 & 1000 \\
\hline
\end{tabular}


根据公式(4) (7)计算关联度：

$\begin{array}{llll}K_{1}\left(v_{1}\right)=-0.0079 & K_{1}\left(v_{2}\right)=-0.4025 & K_{1}\left(v_{3}\right)=-0.3701 & K_{1}\left(v_{4}\right)=-0.2727 \\ K_{2}\left(v_{1}\right)=0.008 & K_{2}\left(v_{2}\right)=-0.2033 & K_{2}\left(v_{3}\right)=-0.1491 & K_{2}\left(v_{4}\right)=0.3333 \\ K_{3}\left(v_{1}\right)=-0.8992 & K_{3}\left(v_{2}\right)=0.195 & K_{3}\left(v_{3}\right)=0.2125 & K_{3}\left(v_{4}\right)=-0.2 \\ K_{4}\left(v_{1}\right)=-0.9899 & K_{4}\left(v_{2}\right)=-0.1403 & K_{4}\left(v_{3}\right)=-0.1491 & K_{4}\left(v_{4}\right)=-0.4667 \\ K_{5}\left(v_{1}\right)=-0.999 & K_{5}\left(v_{2}\right)=-0.3677 & K_{5}\left(v_{3}\right)=-0.3938 & K_{5}\left(v_{4}\right)=-0.6\end{array}$

根据公式 (8) 计算出优化关联度:

$\begin{array}{llll}k_{11}=-0.0079 & k_{12}=-0.4025 & k_{13}=-0.3701 & k_{14}=-0.2727 \\ k_{21}=0.01778 & k_{22}=-0.2033 & k_{23}=-0.1491 & k_{24}=0.0033 \\ k_{31}=-0.8992 & k_{32}=1.95 & k_{33}=2.125 & k_{34}=-0.2 \\ k_{41}=-0.9899 & k_{42}=-0.1403 & k_{43}=-0.1491 & k_{44}=-0.4667 \\ k_{51}=-0.999 & k_{52}=-0.3677 & k_{53}=-0.3938 & k_{54}=-0.6\end{array}$

表4 大桥悬臂施工失效评判矩阵A。

\begin{tabular}{lllllll}
\hline $\boldsymbol{A}_{\mathbf{1}}$ & 评价内容 & $\boldsymbol{a}_{\mathbf{1 1}}$ & $\boldsymbol{a}_{\mathbf{1 2}}$ & $\boldsymbol{a}_{\mathbf{1 3}}$ & $\boldsymbol{a}_{\mathbf{1 4}}$ & $\mathbf{A}$ \\
\hline$a_{11}$ & 悬臂施工失效 & 1 & 2 & 3 & 4 & 0.467 \\
$a_{12}$ & 自然环境 & $1 / 2$ & 1 & 2 & 3 & 0.278 \\
$a_{13}$ & 悬臂施工艺 & $1 / 3$ & $1 / 2$ & 1 & 2 & 0.160 \\
$a_{14}$ & 管理水平 & $1 / 4$ & $1 / 3$ & $1 / 2$ & 1 & 0.095 \\
$\lambda_{\max }=4.030$ & $C_{I}=0.010 \quad C_{R}=0.011<0.1$ & & & & & \\
\hline
\end{tabular}

\section{4. 确认权系数与优度确定}

大桥悬臂施工失效评判矩阵与本文 2.2 节相同, 邀请 相关专家通过 $1 \sim 9$ 标度法对各因素间进行两两比较并赋 值, 构造成判断矩阵后计算权重（见表4）对判断矩阵进 行一致性的检验。

计算得出权重集与其最大特征值。

$$
A=\{0.467 、 0.278 、 0.16 、 0.095\}
$$

$$
C\left[L_{1}\right]=A K\left(L_{1}\right)=A\left[\begin{array}{l}
k_{11} \\
k_{21} \\
k_{31} \\
k_{41}
\end{array}\right]=-0.2007
$$

可 得 $C\left[L_{1}\right]=-0.2007$, 同 理 $C\left[L_{2}\right]=-0.0718 \quad, \quad C\left[L_{3}\right]=0.4432 \quad, \quad C\left[L_{4}\right]=$ $-0.5695, C\left[L_{5}\right]=-0.6888$ 。

对 $L_{j}$ 的优度进行比较知, $C\left[L_{3}\right]=0.4432$ 最大, 悬臂施 工失效可能性为III级, 故该大桥悬臂施工风险为中等风险。

\section{4. 不同模型评价结果对比}

采用文献[14、16、17]的评估方法对本文工程案例数 据进行处理, 所得结果与本文结果对比见表 5 。
本文得到的悬臂施工风险与按文献[17]方法评价 结果有偏差, 主要在于文献[17]方法各项指标权重用 AHP法确定, 导致评价结果随判别矩阵选取的不同而 改变。采用文献[14]和 [16]的方法评估的结果与文中结 论一致, 验证了物元可拓理论应用于悬臂施工安全评 估的有效性。

\section{5. 结论}

本文对某连续刚构桥进行了悬臂施工安全评估,以定 量的数值表示评价结果, 较完整地反映了大桥悬臂施工的 可靠性, 同时验证了物元可拓理论应用于悬臂施工安全评 估是可行和有效的。

采用可靠度理论计算出大桥悬臂施工的失效概率, 再 以可拓理论将实际环境和施工因素的影响以定量的形式 表示。利用优度评价法评判大桥施工各安全等级的优度。

按照优度评价法, $C\left[L_{3}\right]=0.4432$ 最大, 大桥悬臂施 工安全评估结果为中等风险, 综合考虑了失效的不确定性 与模糊性, 同时较为客观地评估了悬臂施工的安全状态。 评估给出了大桥悬臂施工的安全现状, 为避免悬臂施工可 靠性下降以及优化施工悬臂施工管理和施工操作供了可 靠依据。

表5 不同评价方法的结果对比。

\begin{tabular}{lllll}
\hline 评价方法 & 文献[14]方法 & 文献[16]方法 & 文献[17]方法 & 本文方法 \\
\hline 结果 & 中等风险 & 中等风险 & 低等风险 & 中等风险 \\
\hline
\end{tabular}




\section{参考文献}

[1] 穆加宇. 结构可靠性理论在桥梁工程中的应用[D].浙江大 学,2002.

[2] 刘扬,张建仁. 有限元法和退火进化算法相结合分析结构模 糊可靠性[J].工程力学,2002(05):72-77.

[3] 王否. 既有钢筋混凝土桥梁模糊时变可靠性与承载力评估 研究[D].长沙理工大学,2005.

[4] 岐峰军. 基于可靠度理论的刚构桥施工阶段安全性评估[D]. 长安大学,2018.

[5] Caitlyn Davis-McDaniel,Mashrur Chowdhury,Weichiang Pang,Kakan Dey. Fault-Tree Model for Risk Assessment of Bridge Failure: Case Study for Segmental Box Girder Bridges[J]. Journal of Infrastructure Systems,2013,19(3).

[6] 杨春燕, 蔡文. 可拓工程研究 [J]. 中国工程科 学,2000(12):90-96.

[7] 杨雅勋,李子春,李子青,郝宪武. 桥梁状态的区间可拓评估 模型 $[J]$. 交通运输工程学报,2009,9(06):36-41.

[8] 张海,王琳,赵岩,柴旺,孙国龙.基于可拓理论对混凝土梁桥 的可靠性评价 [J]. 沈阳建筑大学学报 (自然科学 版),2013,29(05):868-875.

[9] 张延年,柳成林,李玉兵,郑怡,高飞,沈小俊.基于多指标多级 可拓评价的混凝土梁桥下部结构可靠性评价 [J]. 公 路,2015,60(09):89-95.

[10] 蔡文,杨春燕. 可拓学的基础理论与方法体系 [J].科学通 报,2013,58(13):1190-1199.

[11] 戴树和.工程风险分析技术[M].北京:化学工业出版社,2007.

[12] 周则程,胡万欣,刘玉露,等.群多级可拓理论的高速公路养护 质量评估[J].交通科学与工程,2015,31(02):102-108.

[13] Chen Xiaobo,WU Xin,Dong Cheng.Study on the method of bridge risk matrix decision base on ALARP $[J]$.Highway Engineering,2014,39(1):63-65.

[14] LIU Jun-juan,Wang Wei,Cheng Lin.Interval number fuzzy evaluation based on trapezoid subordinate function[J].Systems Engineering and Electronics,2009,31(2):390-392.

[15] Chen Jiahai. Research on Reliability of Continuous Rigid Frame Bridge Construction State Based on Support Vector Machine[D]. Guangxi University,2018.

[16] Qiao Jiangang, Wang Wei, Li Shixuan.Relative difference function based traffic safety assessment of highway construction[J]. Journal of Chongqing Jiaotong University:Natural Science, 2020,39(07):81-85+99.

[17] Chen Yun,Wu Zhuqing,Liu Qunjian.Risk evaluation of highway engineering project based on the Fuzzy-AHP[J].Journal of Changsha Communications University, 2008(03):44-48. 